CERN/PS 2001-061

\title{
Comparison of Simulation Codes for the Beam Dynamics of Low-Energy Ions
}

\author{
K. Hanke, S. Heising, G. Probert, R. Scrivens
}

\begin{abstract}
Results are presented of the simulation of low-energy ion dynamics with three different codes: KOBRA, IGUN and CPO. To share work between different codes one needs to be confident that they agree. For test cases, good agreement was found between the codes and analytical solutions. Where possible, results have been compared to experimental data from the CERN Laser Ion Source. These simulations are in general agreement with the data of the real beam line.

(c) The following article has been submitted to Review of Scientific Instruments. After it is published, it will be found at http://ojps.aip.org/rsio/.
\end{abstract}

Presented at ICIS2001, Oakland, California, USA

3-7 September 2001

Geneva, Switzerland

October 2001 
Simulation of the extraction and low-energy transport of ions is a complex problem. In the case of the CERN Laser Ions Source (LIS) the beam is space-charge dominated and consists of a variety of charge states. To simulate the transport of beams through electrostatic fields, one starts from a set of measured beam parameters at the entry of the beam line. The result of the tracking depends then on the precision of the measurement, the field solver and the integration routine for the equation of motion of the particles.

The simulation of particle transport in the space-charge domain can be shared between different codes if they have been cross-checked against each other. At CERN, the three codes KOBRA, IGUN and CPO are available. We have cross-checked these codes against each other with simple examples and have compared the results with experimental data available from the CERN LIS. The input beam distribution was identical for all codes. The codes were compared using two tests:

1. Expansion of a space-charge dominated beam in a drift region without external fields;

2. Transport of particles in the electrostatic field of a Gridded Electrostatic Lens (GEL) [1] as used on the CERN LIS (see later description).

\section{Simulation Codes}

\subsection{KOBRA}

KOBRA3-INP [2] is a three-dimensional program for the simulation of steady state problems. The program contains a Vlasov solver, which finds the solution of the Poisson equation and of the particle distribution function via the space-charge map which is then included in further iterations of the Poisson equation.

\subsection{IGUN}

IGUN [3] is a 2D ray-tracing simulation code that is designed for the simulation of ion extraction and transportation in electrostatic and magneto-static systems. The code is based on an electron ray-tracing code (EGUN) with additional modelling of the compensation of the ion space-charge by electrons in a plasma.

\subsection{CPO}

Charged Particle Optics (CPO)[4] is a three-dimensional code that uses the surface charge method. In contrast to the other codes, the electrical fields are determined by charges deposited onto surface elements of the conductors within the volume. A comparison of finite element methods and the surface charge method is given in $[5,6]$.

\section{Space Charge Expansion}

The first example used to cross-check the codes is the space-charge expansion of a beam with given parameters going through a drift space. For this study, a uniform beam of $\mathrm{Ta}^{20+}$ ions was generated with $30 \mathrm{~mm}$ initial diameter and zero emittance ('pencil beam'). The initial kinetic energy was set according to an extraction voltage of $60 \mathrm{kV}$. The drift length was $10 \mathrm{~cm}$ and two beam currents of $60 \mathrm{~mA}$ and $120 \mathrm{~mA}$ were simulated.

The three simulation codes give the output beam parameters shown in Table 1. Note that IGUN provides only two-dimensional results $\left(r, r^{\prime}\right)$ and that the emittances calculated by IGUN are significantly lower compared to other codes. KOBRA and IGUN agree to within $1 \%$ and CPO to within $2 \%$ to the approximate analytical solution of the radius. We are therefore convinced that space-charge is treated correctly in the three codes. 
Table 1: Output beam parameters for space charge expansion computed with the three codes.

\begin{tabular}{|c|cc|cc|cc|}
\hline & \multicolumn{2}{|c|}{ KOBRA } & \multicolumn{2}{c|}{ IGUN } & \multicolumn{2}{c|}{ CPO } \\
\hline & $60 \mathrm{~mA}$ & $120 \mathrm{~mA}$ & $60 \mathrm{~mA}$ & $120 \mathrm{~mA}$ & $60 \mathrm{~mA}$ & $120 \mathrm{~mA}$ \\
\hline$r[\mathrm{~mm}]$ & $17.5^{a}$ & $19.6^{b}$ & 17.3 & 19.6 & 17.2 & 19.2 \\
\hline
\end{tabular}

a) Analytical value is $17.4 \mathrm{~mA}$

b) Analytical value is $19.6 \mathrm{~mA}$

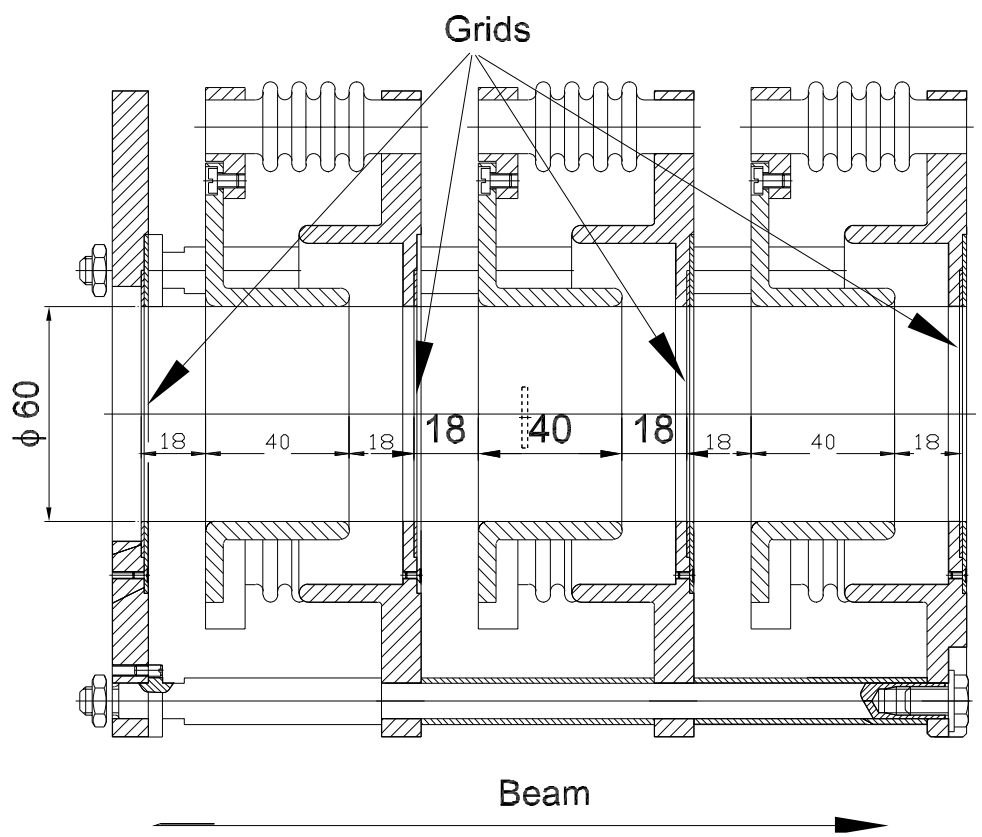

Figure 1: Gridded electrostatic lens LEBT. All dimensions in $\mathrm{mm}$.

\section{Simulation of LEBT}

We will now consider beam transport through an electrostatic focusing element as used in low-energy beam transport (LEBT) lines. The input beam parameters are in general accessible to measurements and therefore known within the measurement precision. The electrostatic field is computed by the field solver of the codes. This is also a possible source of discrepancy between codes.

The gridded electrostatic lens LEBT used on the CERN LIS was chosen as an example. It consists of three long $(40 \mathrm{~mm})$ electrodes at variable potential separated by grids at ground potential. The complete GEL set-up is shown in Fig. 1. It is used to match the beam from the extraction system to the acceptance of the subsequent Radio-Frequency Quadrupole (RFQ).

As a test case to verify the correct beam transfer in the three codes, we have considered one single GEL element at $V=30 \mathrm{kV}$. The geometry used was a $30 \mathrm{~mm}$ drift, one GEL element and a $30 \mathrm{~mm}$ exit drift region. The GEL element consists of a grid at ground potential, a drift of $18 \mathrm{~mm}$, the $40 \mathrm{~mm}$ long electrode at $30 \mathrm{kV}$, a second drift of $18 \mathrm{~mm}$ and a second grid at ground potential. $\mathrm{Ta}^{20+}$ ions at a kinetic energy corresponding to $110 \mathrm{kV}$ extraction were transported. Space-charge effects were suppressed for this test case.

\subsection{Potential on Beam Axis}

The field solvers of the three different codes were compared. The potential map for the given geometry and potentials was computed, and the potential along the $z$ axis was plotted 

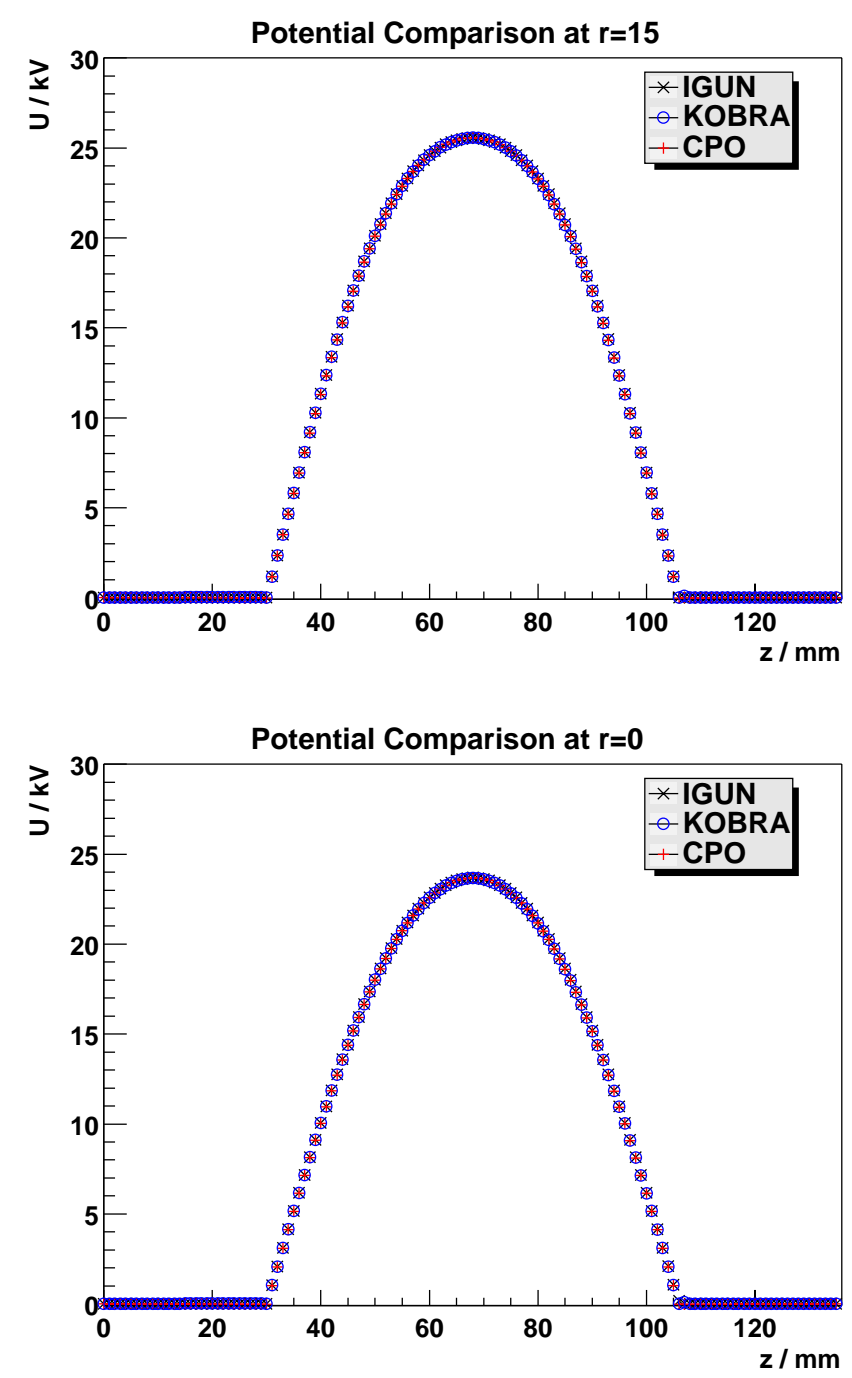

Figure 2: Potential at $r_{x, y}= \pm 15 \mathrm{~mm}$ (upper plot) and $r_{x}=r_{y}=0 \mathrm{~mm}$ (lower plot) as computed by the three codes. No discrepancy is visible.

for the three cases $\left(r_{x}=15 \mathrm{~mm}, r_{y}=0 \mathrm{~mm}\right),\left(r_{x}=0 \mathrm{~mm}, r_{y}=15 \mathrm{~mm}\right)$ and $\left(r_{x}=0 \mathrm{~mm}\right.$, $r_{y}=0 \mathrm{~mm}$ ). The resulting plots are shown in Fig. 2. The difference plot is shown in Fig. 3, where the largest deviation is below $50 \mathrm{~V}$. Therefore it is concluded that the calculation of the potential map is done correctly in all three codes.

\subsection{Transport of Test Rays through GEL}

Single test rays were traced through the potential map. The test rays started at $5 \mathrm{~mm}$, $10 \mathrm{~mm}$ and $15 \mathrm{~mm}$ from the axis in $x$ - and $y$-direction. A plot of the trajectories is shown in Fig. 4, where the rays overlap perfectly.

\subsection{Beam Transport through GEL}

Finally we transported a typical LIS beam with a current of $74 \mathrm{~mA}$ through the three-cell GEL as shown in Fig. 1. The parameters for the initial distribution are taken from a measurement at the entry plane of the GEL. The emittance at $50 \mathrm{~mm}$ after the exit grid as well as the current into a Faraday cup with two input apertures was obtained. The double-aperture cup has two 
rel. Potential Comparison at $r=15$
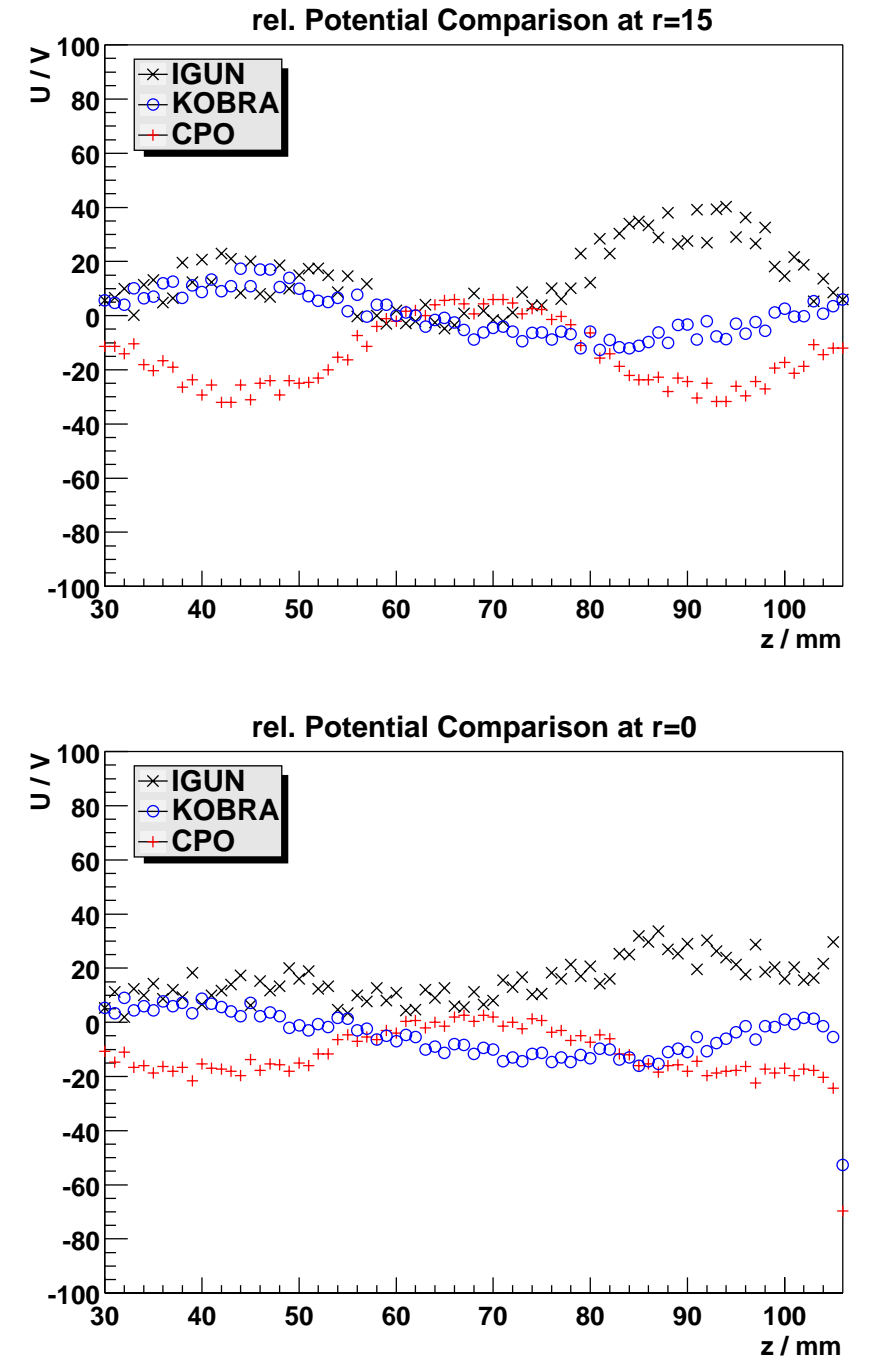

Figure 3: Difference of the potential calculated by the three codes from average potential. The upper plot corresponds to $r_{x, y}= \pm 15 \mathrm{~mm}$ and the lower one to $r=0 \mathrm{~mm}$. 


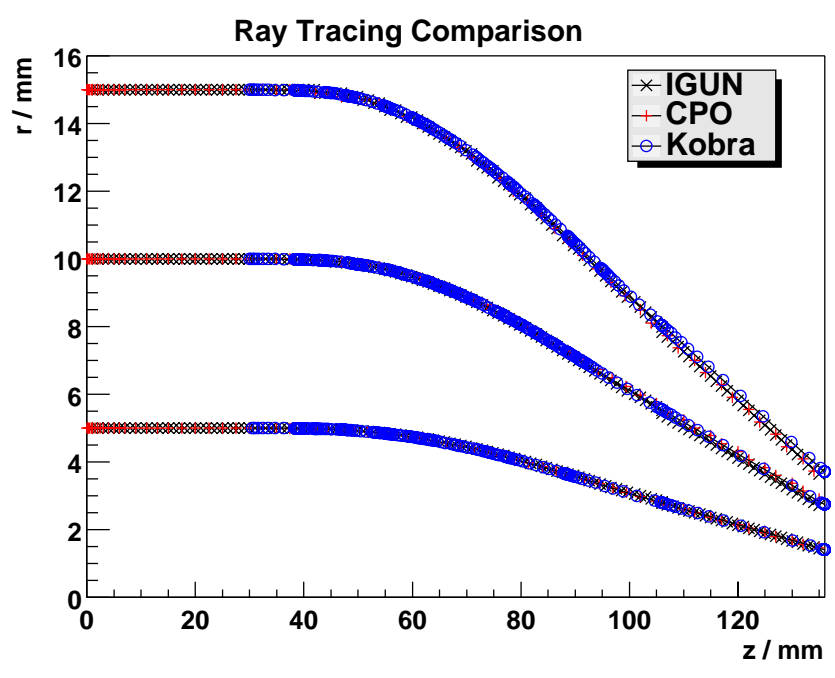

Figure 4: Tracking of test-rays through one cell of the GEL. All codes agree.

Table 2: Output beam parameters for a $240 \pi \mathrm{mm}$ mrad emittance beam through a three-cell GEL. The initial distribution used is based on the measured emittance at the entrance of the GEL. $\varepsilon_{i, f}$ are the initial and final emittances, $I_{D A}$ is the current into a double aperture cup.

\begin{tabular}{|l|cccc|}
\hline & KOBRA & IGUN & CPO & experiment \\
\hline rays & 15000 & 500 & 11000 & \\
$\varepsilon_{4 \mathrm{rms}, \mathrm{i}}[\pi \mathrm{mm} \mathrm{mrad}]$ & 240 & $233 / 241$ & 242 & 242 \\
$\varepsilon_{4 \mathrm{rms}, \mathrm{f}}[\pi \mathrm{mm} \mathrm{mrad}]$ & 420 & $234 / 421$ & 434 & 365 \\
$I_{\mathrm{DA}} / \mathrm{mA}$ & 41 & 36.5 & 41 & 25 \\
$I_{\mathrm{DA}} / \mathrm{mA}\left(\mathrm{scaled}^{c}\right)$ & 33.5 & 29.8 & 33.5 & 25 \\
\hline
\end{tabular}

c) Taking into account losses at the grids.

square apertures of $5.9 \mathrm{~mm}$ spaced $44 \mathrm{~mm}$ apart, with the first aperture positioned $30 \mathrm{~mm}$ downstream of the GEL exit grid. In Table 2, the input and output parameters are summarized for all codes and compared to the experiment. For the 2D-code IGUN the input rays were transformed to polar coordinates $(r, \theta)$ and the result re-mapped to Cartesian coordinates. Since in IGUN all problems are radially symmetric, the apertures represent circles and hence the current was scaled by $\frac{4}{\pi}$ for comparison with a square aperture. IGUN uses only 500 rays which leads to different emittances in $x$ and $y$. A similar behaviour was seen with CPO when only up to 1000 rays could be used.

\section{Conclusion}

Three codes for simulation of low-energy ion dynamics were compared to each other as well as to known solutions and experimental data. The codes reproduce an approximate analytical solution for space-charge calculations to within $2 \%$. Differences of the potentials from the field solvers are in the order of $10^{-3}$ of the maximum voltage. Single rays traced through a test case coincide.

When simulating the low-energy line of the CERN LIS, the three codes exhibit good agreement. However, comparison with the experimental data shows an underestimation of the emittance by $20 \%$ and an overestimation of the transmitted current by $30 \%$.

The simulations presented here do not include the following characteristics of the real 
line: (i) variation of the beam parameters during the current pulse; (ii) more accurate representation of the GEL structure (including for example the grid wires); (iii) space-charge compensation. Furthermore, the average beam parameters include experimental errors and the effect of the measurement equipment on the beam itself. Further simulations can begin by taking these processes into account.

The characteristics of LIS (current, emittance, extraction geometries) change with different laser configurations and target materials. The good agreement between different codes and experiments shows that with these tools the estimation of source parameters within acceptable tolerances is possible, limiting tedious and costly experiments.

\section{References}

[1] P. Ostroumov, LIS LEBT on the base of electrostatic gridded lenses, unpublished.

[2] P. Spädtke, KOBRA3-INP User Manual, version 3.39 (2000).

[3] R. Becker, W. B. Herrmannsfeldt, IGUN - A Program for the Simulation of Positive Ion Extraction including Magnetic Fields, Rev. Sci. Instrum. 63 (4), p.2756-2761 (1992).

[4] Welcome to CPO, Help file for the CPO package, version 3.1e (2001).

[5] D. Cubric, B. Lencova and F. H. Read, Comparison of the Finite Difference, Finite Element and Surface Charge Methods for electrostatic charged particle optics, Electron Microscopy and Analysis 1997, Institute of Physics Conference Series 153, 91-94 (1997).

[6] D. Cubric, B. Lencova, F. H. Read, J. Zlamal, Comparison of the Finite Difference, Finite Element and Surface Charge Methods, Nucl. Instr. Meth. (1999). 